\title{
Trigger factors in childhood migraine: a clinic-based study from Eastern India
}

\author{
Ambar Chakravarty • A. Mukherjee • \\ D. Roy
}

Received: 20 May 2009/ Accepted: 29 July 2009/Published online: 25 August 2009

(C) Springer-Verlag 2009

\begin{abstract}
Literature on triggers of childhood migraine is sparse. This study was carried out in 200 children (715 years) with migraine from a metropolitan city in Eastern India, both retrospectively and prospectively, with headache diaries to note the incidence of various triggers. In the retrospective study, triggers could be identified in $94 \%$ of subjects while in $100 \%$ of children in the prospective part of the study more than one trigger could be identified. Most migraine triggers identified were environmental (sun exposure, hot humid weather, smoke and noise) and stress related (school stress mostly). Quite often these operated concurrently to precipitate individual migraine attacks.
\end{abstract}

Keywords Childhood migraine $\cdot$ Migraine triggers

\section{Introduction}

A migraine trigger is any factor that on exposure or withdrawal leads to the development of an acute migraine attack in a susceptible individual. Triggers may be categorised as behavioral, environmental, infections, dietary, chemical or hormonal. Triggers need to be differentiated from aggravating factors. Triggers are not universal. Moreover, the presence of a trigger factor does not always precipitate an attack in the same individual. This suggests a multifactorial trigger mechanism for individual attacks. Given the fact that migraine is essentially a neurovascular phenomenon, and cerebral cortical hyper excitability a core feature (a concept first postulated by Sir William Gowers)

A. Chakravarty $(\bowtie) \cdot$ A. Mukherjee $\cdot$ D. Roy

Department of Neurology, Vivekananda Institute of Medical

Sciences, 1E 1202, Avishikta II, Calcutta 700 078, India

e-mail: saschakra@yahoo.com
[1], it appears hardly surprising that the already sensitised brain responds rapidly to internal and external triggers. Many studies of these triggers have been published but mostly in relation to adult migraine. A critical analysis of triggers has been made by Martin and Behbehari [2] in 2001 and by Davidoff in 2002 [3]. Kelman [4] reported on triggers (but again in adults) on a large clinic-based sample of migraneurs recently. In comparison, literature on triggers for childhood migraine is sparse [5-8] although Winner et al. [9] provide a long list of identifiable triggers of migraine in childhood and adolescents. There appears to be no published report of triggers in childhood migraine from any South-East Asian country where climatic, ethnic, dietary and socio-cultural factors are vastly different from those in the Western hemisphere. Furthermore, childhood migraine is significantly different from adult migraine in various aspects including sex bias [5], duration [10] and site of pain localisation [11].

In this study, we report our observations on migraine triggers in 200 children with migraine who had been studied in our department both retrospectively and prospectively. The retrospective part of the study had been part of a large study on migraine pain location in children and adults, the results of which had already been published [12].

\section{Materials and methods}

This study was carried out between September 2003 and December 2005 at the neurology out-patients' department (tertiary care centre) of a large general hospital in the city of Calcutta in eastern India. The department generally looks after children from age 7 years and above, younger children being looked after by the paediatricians. The 
hospital mostly caters to middle class people in the southern part of the city. This study, which is actually a part of a previous study reported earlier on migraine pain location in children [12], was carried out in 200 consecutive children with migraine (diagnosed on the basis of ICHD II criteria as applied to children) [10], who were selected out of a total of 262 subjects depending on the children's and their parents consistency in responses to our semi structured questionnaire which included questions relating to various aspects of migraine including pain location, precipitating, aggravating and relieving factors as also of family history on the occurrence of migraine or epileptic seizures. All these subjects had been having at least two attacks of migraine per month for the previous 6 months.

\section{Retrospective study}

At the time of presentation, the children along with at least one of the parents (generally of good intelligence and having basic high school or university education) were evaluated by one of the authors to confirm diagnosis of migraine and exclude secondary headache disorders; then by a trainee for filling up the questionnaire and then again by one of the authors to verify and validate the responses. All the questionnaire sheets were subsequently scrutinised by the corresponding author to clarify any vagueness in the forms from separate interviews with the parents. As regards migraine triggers, a long list of items (Table 1) was prepared based on the lists provided by Winner et al. [9] and others $[2,3]$ and our previous experience with common and uncommon precipitating factors in migraine (children and adults) in our institution keeping Indian climatic, dietary, educational system and socio-cultural factors in mind. Parents were asked to tick only those items which they considered 'definite' in precipitating migraine headaches in their children. As mentioned earlier, any vagueness in responses was clarified through separate interviews by the corresponding author at the end of the first phase of the study.

\section{Prospective study}

For the purpose of the prospective study, 197 children with migraine without aura were asked to be followed up in the clinic for the next 3 months at 6 weeks intervals. The three children with migraine with aura were excluded. The parents were provided with diaries to note brief clinical details of any headache spells especially their nature, duration, severity (Scale 1-7), associated features, and note the presence of any precipitating factor/factors from the lists they were already asked to answer on during the retrospective part of the study. The pain scale mentioned
Table 1 Migraine triggers in questionnaire
A. Environmental/climatic
1. Sunlight
2. Hot and humid climate
3. Cold (air conditioner)
4. Noise

(a) Environmental

(b) Loud speakers/loud music

5. Smoke
(a) Auto emissions
(b) Cigarettes
6. Crowded place
B. Visual stimuli
1. School book reading
2. Recreational reading
3. Bright light (other than sun)
4. TV watching
C. Stresses
1. School stress
2. Extracurricular activity related
3. Family stress
4. Bereavement
D. Physical exercise (games)
E. Dietary
1. Missing meals
2. Religious fasting
3. Dark chocolates
4. Pizza
5. Chinese food
6. Caffeinated drinks
F. Car/bus journeys (other than to school)
G. Behavioral
1. Shouting
2. Being scolded
H. Hormonal
1. Menstruation
I. Others (perfumes, head trauma, G I Upset)
J. No Triggers

had not been validated formally but had been in use by us for long and found to be quite useful. During this phase, no prophylactic drug therapy was prescribed, and only a general advice regarding avoidance of noted triggers was given. The data collection was completed by April 2006 and 186 children (drop out 11) and their parents completed a 3 month's follow up.

All the headache spells were assessed (by one of the three authors) based on their description in the diaries, and only those fulfilling the diagnostic criteria of migraine were noted. The total number of headache spells was calculated 
as also the number of headache spells precipitated by any particular trigger was noted and summed up. Any vague responses in relation to headache phenomenology or triggers were discarded.

\section{Results}

\section{Demography}

Out of the 200 subjects, initially evaluated, there were 118 males and 82 females (M:F 1:0.69) and their ages varied from 7 to 15 years (mean 11.8 years; SD 0.8). Duration of migraine ranged from6 months to 4 years. (mean 1.6 year). Most $(n=197$, i.e. $98.5 \%)$ had migraine without aura (1.1) and only three $(1.5 \%)$ had migraine with typical aura (1.2.1). Out of the 186 children followed up at 3 months, there were 108 boys and 78 girls. The sex bias noted in the study had been noted and reported by using our previous study on chronic daily headaches in children and mentioned later in this article.

\section{Retrospective study}

Migraine triggers identified in the retrospective study based on responses to interviews of subjects and their parents as per the study questionnaire on migraine triggers are detailed in Table 2. Attempts at further detailing (e.g. approximate percentage or relation to severity of attack) on this issue on most occasions led to vague responses and hence abandoned. Among the 200 subjects, one or more triggers could be identified in 188 subjects (i.e. 94\%). The three children with migraine with aura did not have any trigger.

\section{Prospective study}

The results are depicted under two categories. Table 3 summarises number of headache spells precipitated by any particular trigger at 3 months' follow up in 186 subjects. Triggers could be identified in all the 186 subjects $(100 \%)$.

Table 4 lists triggers generally found to be associated with severe (5 or more on a scale of 7 ) migraine attacks.

\section{Summary observations}

Results of both the retrospective and 3 months' prospective study reveal that in Indian children in a metropolis in the eastern part of the country, major triggers of migraine headache (as also often for severe headache spells) include-sun exposure, hot and humid climate, environmental noise, crowded place and school-related stress.
Table 2 Incidence of triggers in retrospective study $N=200$

\begin{tabular}{|c|c|c|}
\hline Trigger & $n$ & $\%$ \\
\hline \multicolumn{3}{|l|}{ A. Environmental/climatic } \\
\hline 1. Sunlight & 188 & 94 \\
\hline 2. Hot and humid climate & 188 & 94 \\
\hline 3. Cold (air conditioner) & 2 & 0.5 \\
\hline \multicolumn{3}{|l|}{ 4. Noise } \\
\hline (a) Environmental & 156 & 78 \\
\hline (b) Loud speakers/loud music & 134 & 67 \\
\hline \multicolumn{3}{|l|}{ 5. Smoke } \\
\hline (a) Auto emission & 83 & 41 \\
\hline (b) Cigarettes & 7 & 3.5 \\
\hline 6. Crowded place & 156 & 78 \\
\hline \multicolumn{3}{|l|}{ B. Visual stimuli } \\
\hline 1. School book reading & 105 & 52.5 \\
\hline 2. Recreational reading & 11 & 5.5 \\
\hline 3. Bright light (other than sun) & 58 & 29 \\
\hline 4. TV watching & 17 & 8.5 \\
\hline \multicolumn{3}{|l|}{ C. Stresses } \\
\hline 1. School stress & 157 & 78.5 \\
\hline 2. Extracurricular activity related & 37 & 18.5 \\
\hline 3. Family stress & 29 & 14.5 \\
\hline 4. Bereavement & 2 & 1 \\
\hline D. Physical exercise & 27 & 13.5 \\
\hline \multicolumn{3}{|l|}{ E. Dietary } \\
\hline 1. Missing meals & 63 & 31.5 \\
\hline 2. Religious fasting* & 11 & 13.4 \\
\hline 3. Dark chocolate & 3 & 1.5 \\
\hline 4. Pizza & 2 & 1 \\
\hline 5. Chinese food & 7 & 3.5 \\
\hline 6. Caffeinated drinks & 0 & 0 \\
\hline F. Car/bus journeys (other than school) & 33 & 16.5 \\
\hline \multicolumn{3}{|l|}{ G. Behavioral } \\
\hline 1. Shouting & 29 & 14.5 \\
\hline 2. Being scolded & 13 & 6.5 \\
\hline \multicolumn{3}{|l|}{ H. Hormonal } \\
\hline 1. Menstruation* & 3 & 3.7 \\
\hline \multicolumn{3}{|l|}{ I. Others } \\
\hline (Perfumes, head trauma, GI upset) & 9 & 4.5 \\
\hline J No trigger & 12 & 12 \\
\hline
\end{tabular}

$*$ In girls $(n=82 ;$ menstruating $=36)$

School book reading was found to be a trigger in over $50 \%$ of subjects. Dietary factors seem to be much less operational though, in some older girls, religious fasting precipitated severe migraine attacks. It appears from the results in Table 2 and 3 that many of the environmental factors mentioned above act concurrently to precipitate migraine attacks. Also of interest to note is that some parents who denied having any trigger in the retrospective 
Table 3 Incidence of triggers in prospective study (at 3 months) $N=186$ No. of migraine spells $=1,563$ (i.e. 2.8 Spells/subject/ month)

\begin{tabular}{l} 
Trigger \\
\hline A. Environmental/climatic \\
1. Sunlight \\
2. Hot and humid climate \\
3. Cold (air conditioner) \\
4. Noise
\end{tabular}

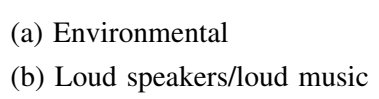

5. Smoke

(a) Auto emission

(b) Cigarettes

6. Crowded place

B. Visual stimuli

1. School book reading

2. Recreational reading

3. Bright light (other than sun)

4. TV watching

C. Stresses

1. School stress

2. Extracurricular activity related

3. Family stress

4. Bereavement

D Physical exercise

E. Dietary

1. Missing meals

2. Religious fasting*

3. Dark chocolate

4. Pizza

5. Chinese food

6. Caffeinated drinks

F. Car/bus journeys (other than school)

G. Behavioral

1. Shouting

2. Being scolded

H. Hormonal

1. Menstruation*

I. Others

(Perfumes, head trauma, GI upset)

Number of school days lost: 440 (i.e. 0.8 days/subject/month)

* In girls $(N=78$; no. of headache spells in 36 menstruating girls: 288)

study, mentioned of triggers in the prospective study when they were provided with headache diaries. Thus, at the end of the 3 months' prospective follow up some (almost always more than one) trigger or other could be identified in $100 \%$ of children.

$\begin{array}{ll}\begin{array}{l}\text { Migraine spells } \\ n=1,563\end{array} & \% \\ & \\ 1,312 & 83.9 \\ 1,325 & 84.8 \\ 0 & 0\end{array}$

$1,085 \quad 69.4$

59.1

36.5

0.77

54.4

37.6

5.9

18.6

4.5

88.9

11.8

14.3

0

11.6

13.4

0.2

0.13

0

0.32

0

0.2

13.8

3.3

2.4

$N=288$
Table 4 Triggers associated with severe migraine headache (severity $\geq 5$ on scale of 7 )

\section{Discussion}

This study appears to be the only one reported so far from a tropical developing country especially in South East Asia on migraine triggers in children. Headache treatment on the whole, is somewhat different in India than in the west in the sense that the entire environment is different, doctors in general are hard pressed for time due to acute patient load, paucity of neurologists vis-àvis population rise and patients in general are reluctant to use drugs for their ailments. In relation to migraine, the last factor is somewhat beneficial as we tend to see lesser number of analgesic overuse headaches [13], but on the other hand, this imposes greater responsibility (and longer time) on doctors (especially neurologists) to impress on patients regarding the various modes of nonpharmacologic management. Here lies the importance of identifying trigger factors as avoidance of 'controllable' triggers may be a corner stone in prophylactic nonpharmacologic management of migraine. Controlled studies in this respect are, however, sparse [2], and most triggers are often environmental, social or behavioral and difficult to avoid.

In order to appreciate the uniqueness of migraine triggers in India in middle class people (especially children), one needs to look at the overall scenario. The climate is hot (especially in eastern India) for most time of the year, winter becoming gradually practically non-existent as a result of global warming; there is plenty of sunshine almost all round the year but humidity remains very high most of the times. Air pollution is high with dust and auto-emitting smoke mostly in urban areas like Calcutta. Children grow up in this atmosphere and by school-going age, they face much greater problems. Parents' academic expectations are always very high in middle class societies - so also their expectation for their children to excel in all the activities such as sports and arts. Academic competition is increasing day by day, and parents always dream to send their children to the very best of schools and universities. A typical school-going child's day starts early in the morning with tutoring by parents, followed by rushing to school-completing home works on the way, continuous attendance in classes for long hours with practically no breaks, being escorted to games field/swimming by the mother or 
attendant in the early afternoon followed by attending classes in fine arts and then going for private tutorials. On returning home late in the evening, tutoring by parents await them. The children are exhausted so also are their parents, both of whom often happened to be working. Relaxation is rare, and even weekend chores are not much different. Exhausted and irritated parents do at times fight, making the overall nuclear family atmosphere rather stressful for these young children. It is in this background that migraine triggers in children in India needs discussion.

Exposure to sunlight (for $>30 \mathrm{~min}$ ) and a hot humid weather (even while at home) have been common triggers. Sun exposure and hot weather are recognised triggers in western studies. However, in India, these appear more frequent with apparently more intense headaches on very hot days as revealed in diaries. On the other hand, cold weather which is recognised as a precipitant in the west is an uncommon trigger in our study-probably because the average temperature rarely drops below $15^{\circ} \mathrm{C}$ in winter.

Exposure to auto-emitting smoke has been a trigger in many and here in addition to the smoke itself, the odour of burnt fuel (usually diesel in public transport vehicles) adds to the malady.

It is rather difficult to clearly identify one particular trigger when a child gets a migraine attack in the afternoon on returning from school. He/she is exhausted, exposed to sun and a hot humid weather and also to crowd in the public transport vehicle which is often noisy with blaring car and bus horns, and he/she had to bear the school stress as well. It is obvious that several triggers act concurrently to precipitate individual migraine spells.

Reading and migraine precipitation is a unique feature highlighted in the study. School book reading was identified as a trigger in over 50\% children in the retrospective study and precipitated over a third of headache spells in the 3 months of prospective follow-up with diaries. Detailed interview of parents convinced us on the migrainous nature of these headache spells. Ocular causes including so called 'eye strain' headaches were carefully excluded in all. Interestingly, headaches rarely occurred during recreational reading (story books, comics). This suggests an element of 'stress' in the genesis of the headaches and not solely visual stimulation. However, many of these children also had migraine precipitated by bright light including sun exposure. Therefore, we feel, although visual stimulation may have a role in the genesis of the headache induced by reading, the final clinical expression is determined by the associated stress factor. Stress thus appears to be a determinant factor in many children with migraine, and the effect of school and parental stress, as briefly outlined early, need not be further elaborated. Proper counselling of children and their parents seems to be the only reasonable solution.
Dietary factors have been much stressed in western literature but seem to be an uncommon trigger in Indian children. Migraine precipitated by religious fasting is quite common in older Indian women and was noted in a few older girls in the retrospective part of the study. Minor head trauma precipitated severe migraine in two children and gastrointestinal disturbance occasionally precipitated migraine attacks.

Menstruation had been noted as a trigger in relatively smaller number of female subjects in the study. While our results of the study of triggers in subjects older than 15 years are being analysed, it is our impression that on the whole menstrual trigger of migraine headaches is less common in India than in the West.

In conclusion, environmental factors and academic stress seem to be major triggers for occurrence of most migraine attacks in Indian children. Also, several factors operate concurrently in the precipitation of individual attack on most instances. What seems puzzling is the fact that although children are exposed to these triggers (environmental and stress related) days after days all round the year, why do they get migraine only on some days in a month? In fact chronic daily headaches (CDHs) are relatively uncommon in Indian children [13] compared to adults [14]. It is likely that some form of adaptive mechanism in cortical excitability is in operation.

\section{Study limitations}

1. This is a clinic-based study and not population based.

2. Metropolitan setting is different from rural setting and triggers might differ.

3. Prospective part of the study had been for a relatively short period ( 3 months) although we could record over 1,500 headache spells.

4. Relative importance of individual environmental and stress factors could not be assessed, as it seemed, they operate concurrently quite often.

5. There may be a little flaw in the prospective part of the study in the sense that $100 \%$ of subjects identified a trigger. Such a flaw is inevitable in questionnairebased study such as this where environmental conditions remain same, and it is tempting for responders to associate such constant factors with an event as no objective parameter can be used. Thus, some degree of overestimation in relation to environmental triggers of migraine is possible. Establishing role of environmental factors as triggers is fraught with some problems as had been addressed to earlier by Wober et al. [15].

6. It would have been of interest to compare trigger factors noted in the study ( $7-15$ years) to those possible in children below 7 years of age and subjects above 15 years of age. While data on triggers in adult 
subjects with migraine are being analysed, we have no data in relation to children below 7 years of age as the Department only caters to subjects above 7 years of age as had been mentioned earlier.

\section{Conflict of interest None.}

\section{References}

1. Gowers WR (1907) The border-land of epilepsy: migraine. Old Hickory Bookshop, Philadelphia, pp 76-104

2. Martin VT, Behbehari MM (2001) Towards a rational understanding of migraine trigger factors. Med Clin N Am 85:911-941

3. Davidoff RA (2002) Initiators, precipitants and triggers. In: Davidoff RA (ed) Migraine: manifestations, pathogenesis and management, 2nd edn. Oxford Press, New York, pp 90-119

4. Kelman L (2007) The triggers or precipitants of acute migraine attack. Cephalalgia 27:394-402

5. Linder SL, Winner P (2001) Pediatric headache. Med Clin N Am 85:1037-1053

6. Millichap JG, Yee MM (2003) The dust factor in pediatric and adolescent migraine. Pediatr Neurol 28:9-15
7. Mack KJ (2006) Episodic and chronic migraine in children. Semin Neurol 26:223-231

8. Lewis DW (2009) Pediatric migraine. Neurol Clin 27:481-501

9. Winner P, Linder SL, Wasiewski WW (2001) Pharmacologic treatment of headache. In: Winner P, Rothner AD (eds) Headache in children and adolescents. B C Decker, Hamilton, pp 87-115

10. Headache Classification Subcommittee of the International Headache Society (2004) The international classification of headache disorders, 2nd edn. Cephalalgia 24(Suppl 1):8-160

11. Chakravarty A, Mukherjee A, Roy D (2008) Migraine pain location: how do children differ from adults? J Headache Pain 9:375-379

12. Chakravarty A, Mukherjee A, Roy D (2007) Migraine pain location at onset and during established headaches in children and adolescents: a clinic based study from Eastern India. Cephalalgia 27:1109-1114

13. Chakravarty A (2005) Chronic daily headaches in children and adolescents: a clinic based study from India. Cephalalgia 25:795800

14. Chakravarty A (2003) Chronic daily headaches in India. Cephalalgia 23:348-353

15. Wober C, Holzhammer J, Zeitlhofer J, Wesseley P, Wober-Bingol C (2006) Trigger factors of migraine and tension type headache: experience and knowledge of the patients. J Headache Pain 7:188-195 